\title{
Pump-probe scheme for optical coherence tomography using indocyanine green mixed with albumin or human plasma
}

\author{
${ }^{1}$ Zahid Yaqoob, ${ }^{2}$ Jeffrey P. Fingler, ${ }^{3}$ Stéphane Lintner, ${ }^{4}$ Brian E. Applegate, ${ }^{1}$ Changhuei Yang \\ ${ }^{1}$ Electrical Engineering, 341 Moore, California Institute of Technology, Pasadena, CA 91125 \\ Tel.1626)395-4711,Fax (626395-8952,zyaqoob@caltech.edu; chyang@caltech.edu \\ ${ }^{2}$ Applied Physics, MC 114-36, California Institute of Technology, Pasadena, CA 91125 \\ Tel. (626) 395-5815, fingler@its.caltech.edu \\ ${ }^{3}$ Applied \& Computational Mathematics, Guggenheim 226, California Institute of Technology, Pasadena, CA 91125 \\ Tel. (626)395-4746, lintner@acm.caltech.edu \\ ${ }^{4}$ Dept. of Biomedical Engineering, 136 Hudson Hall, Duke University, Durham, NC 27708 \\ Tel. (919) 660-2475, Fax (919) 660-2476, brian.applegate@duke.edu
}

\begin{abstract}
Use of indocyanine green (ICG) in a pump-probe scheme for OCT is proposed. The study illustrates that ICG in protein solution shows unusual pump-probe imaging potential, indicating its usefulness as a contrast agent for OCT.

(C)2005 Optical Society of America

OCIS codes: (110.4500) Optical coherence tomography, (170.3880) Medical and biological imaging
\end{abstract}

\section{Introduction}

Optical coherence tomography [1] (OCT) is an excellent technique for high resolution imaging of biological samples to a depth of few millimeters. Various approaches such as Doppler [2] and birefringence [3] have been adopted to add to the functionality of OCT. Recent developments of a new OCT imaging modality known as molecular contrast optical coherence tomography (MCOCT) [4,5] represent different and exciting type of functional OCT methods, in which the major advantages of fluorescence microscopy (chemical contrast detection and imaging capability) and OCT (higher spatial resolution and depth penetration) are combined. The sodium iodide salt of indocyanine green (ICG-Nal) is an FDA approved fluorescent dye used in medical diagnosis and photodynamic therapy. Recently, ICG-NaI was used as a contrast agent in an MCOCT implementation in which contrast agent's distribution inside the sample was determined from agent's spectral differential absorption [6]. This paper proposes and demonstrates, for the first time, an MCOCT implementation based on a pump-probe scheme [4] which uses ICG-NaI as the contrast agent.

\section{ICG-NaI Dynamics}

ICG-NaI has a well defined absorption spectrum [see Fig. 1(a)] that peaks at $\sim 790 \mathrm{~nm}$ when mixed in water. For higher concentrations, the absorption peak is blue-shifted due to the formation of dimers [7]. However, when mixed with albumin or human plasma, the ICG-NaI absorption spectrum red shifts and peaks at $\sim 810 \mathrm{~nm}$. This indicates a strong interaction between ICG-NaI and plasma proteins [7]. The electronic manifold of ICG-NaI shown in Fig. 1(b) indicates that a pump-probe scheme can be devised that pumps and probes the same transitions. If we pump the $1 \rightarrow 2$ transition at $\lambda_{\text {pump }}$, population $\mathrm{N}_{3}$ and $\mathrm{N}_{4}$ will build up in states 3 and 4 , respectively. The lifetime of (Pisomer) state 4 is $\geq 10,000 \mu \mathrm{s}$ and is much longer than the lifetime of the (Triplet) state 3 . Note that this long lifetime exists only for ICG in a protein solution [7]. Thus, if we record an OCT image at early times, $t_{1}$ and at time after the system has come to a steady state, $t_{2}$, the difference image owing to the differential absorption of ICG-NaI as a result of

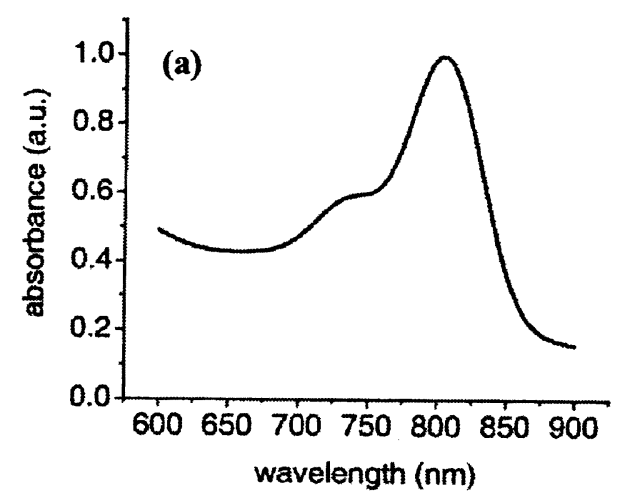
population build up in state 4 will reveal the distribution of the ICG

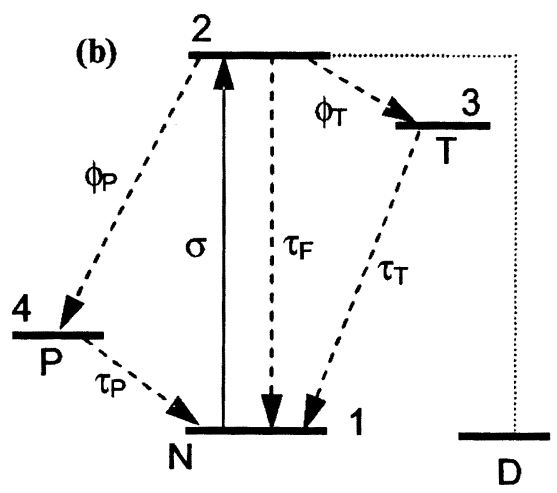

Fig. 1. (a) Absorption spectrum of ICG-NaI. (b) Schematic diagram of the levels of interest in the electronic manifold of ICG-NaI. inside the sample. The corresponding differential equations for population build up in level 4 can be written as [7] 


$$
\frac{\partial N_{4}}{\partial t^{\prime}}=\frac{I_{\mathrm{L}}}{I_{\text {sat } \mathrm{P}} \tau_{\mathrm{P}}}\left(N_{0}-N_{3}-N_{6}\right)-\frac{N_{4}}{\tau_{\mathrm{P}}}\left(1+\frac{I_{\mathrm{L}}}{I_{\text {sat } \mathrm{P}}}\right) ; \quad I_{\text {sat } \mathrm{P}}=\frac{h v_{\mathrm{L}}}{\sigma \phi_{\mathrm{P}} \tau_{\mathrm{P}}},
$$

where $I_{\mathrm{L}}$ and $v_{\mathrm{L}}$ represent the intensity and frequency, respectively, of the pump laser, $\tau_{\mathrm{P}}$ is the lifetime corresponding to state $4, \phi_{\mathrm{P}}$ is the fractional population transferred from state 2 to 4 , and $I_{\text {sat, } \mathrm{P}}$ is the saturation intensity. A pump-probe setup was realized to study the dynamics of ICG-NaI. Light from a Ti:sapphire laser is split into pump and probe beams using and 90/10 beam splitter. An acousto-optic modulator (AOM) placed in the path of pump beam is used as a beam chopper. The +1 order diffracted beam is deflected by a $50 / 50$ splitter and focused on a 500 microns path length flow cuvette, containing $200 \mu \mathrm{M}$ ICG-NaI solution in $1 \%$ albumin, via $11 \mathrm{~mm}$ focal length (FL) lens $\mathrm{L}_{1}$. The probe beam passes through a filter wheel and is focused at the same position inside the cuvette via another $11 \mathrm{~mm}$ FL lens $\mathrm{L}_{2}$. The $140 \mathrm{MHz}$ RF drive signal fed into the AOM is amplitude modulated by a square wave signal of variable duty cycle. When the AOM is OFF (no pump beam), the probe beam (being very weak) is mostly absorbed by the ICG-NaI solution. However, as the AOM is turned ON via the electronic control of the RF drive signal, the population $\mathrm{N}_{4}$ in state 4 starts building up, thus bleaching the ICG-NaI solution. As the dye bleaches, the amount of probe light passing through the ICG-NaI and reaching a high-speed detector via the 50/50 splitter starts increasing. As the population $N_{3}$ and $N_{6}$ are very small compared to $N_{0}$, one can safely assume $N_{6}=N_{3} \cong 0$, to determine the relative population $\mathrm{N}_{4} / \mathrm{N}_{0}$ under steady-state condition as:

$$
\frac{N_{4}}{N_{0}}=\frac{I_{\mathrm{L}}}{I_{\text {sa }, \mathrm{P}}}\left(1+\frac{I_{\mathrm{L}}}{I_{\text {sa }, \mathrm{P}}}\right)^{-1}
$$

Using $I_{\text {sat, }}=3 \times 10^{3} \mathrm{~W} / \mathrm{cm}^{2}$ and pump beam power of $5 \mathrm{~mW}$, steady-state value for relative population $\mathrm{N}_{4} / \mathrm{N}_{0}$ can be estimated as $74.5 \%$. The temporal build up of relative population $\mathrm{N}_{4} / \mathrm{N}_{0}$ can be determined from the photo-detector voltage signal as:

$$
\frac{N_{4}(t)}{N_{0}}=\frac{1}{\sigma N_{0} L} \ln \left[\frac{V(t)}{V(0)}\right]
$$

where $\sigma$ is ground state absorption cross section of the ICG and $L$ is the path length of the cuvette. Figure 2 shows the photo-detector voltage signal versus time as the AOM is turned ON and OFF at the set duty cycle. Using $\sigma=$ $8.2 \times 10^{-16} \mathrm{~cm}^{2}$, the relative population $\mathrm{N}_{4} / \mathrm{N}_{0}$ after $2.5 \mathrm{sec}$ pump beam $\mathrm{ON}$ duration is estimated by Eq. (3) as $9.5 \%$. The discrepancy may be attributed to limited $(2.5 \mathrm{sec})$ pump beam duration, photodegradation, and ICG flow (convection) inside the cuvette. This is also supported by the fact that both bleaching as well as de-bleaching data (shown in Fig. 2) can only be fitted using more than one exponentials.
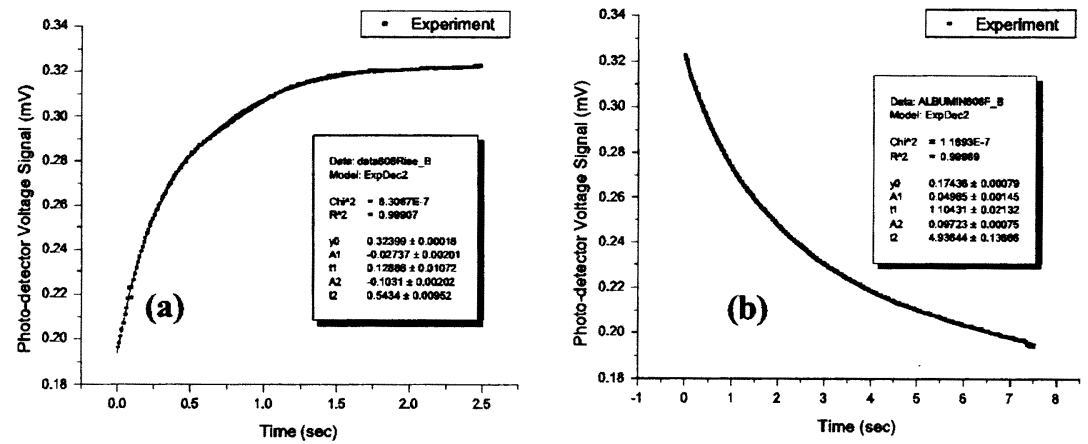

Fig. 2. Photo-detector voltage signals versus time corresponding to (a) bleaching and (b) debleaching of the ICG-NaI solution in $1 \%$ albumin.

\section{Molecular Contrast Optical Coherence Tomography Setup}

The spectral domain fiber-optic MCOCT setup, shown Fig. 3(a), uses two $2 \times 2$ fiber couplers. Light from a modelocked Ti:sapphire (12 nm FWHM) is coupled into a standard $5 / 125 \mu \mathrm{m}$ single mode fiber (SMF) for spectrum broadening. The broadband probe light $(\sim 45 \mathrm{~nm}$ FWHM centered at $780 \mathrm{~nm})$ from the SMF fiber is coupled into the first coupler via a fiber-optic attenuator (FOA) to control the amount of light reaching the sample. One of the output ports of the first fiber coupler is connected to an optical spectrum analyzer to monitor the spectrum of the source whereas the other is connected to the second fiber coupler. The other input port of the second coupler is connected to a fiber-coupled pump laser operating at $806.25 \mathrm{~nm}$. The output arms of the second coupler make the reference and sample arms. A $1 \mathrm{~mm}$ path length cuvette filled with $200 \mu \mathrm{M}$ ICG-NaI solution in $1 \%$ albumin is used as the sample. The polarization controller (PC) in the sample arm corrects for the birefringence artifacts in the sample and/or reference arms. Light from the reference and sample is collected back via the same optics and detected by Ocean Optics, Inc. fiber-coupled spectrometer (HR4000) connected to the input port of the first fiber-coupler. A personal computer (PC) is used to both control the pump laser as well as acquire data from the spectrometer. 

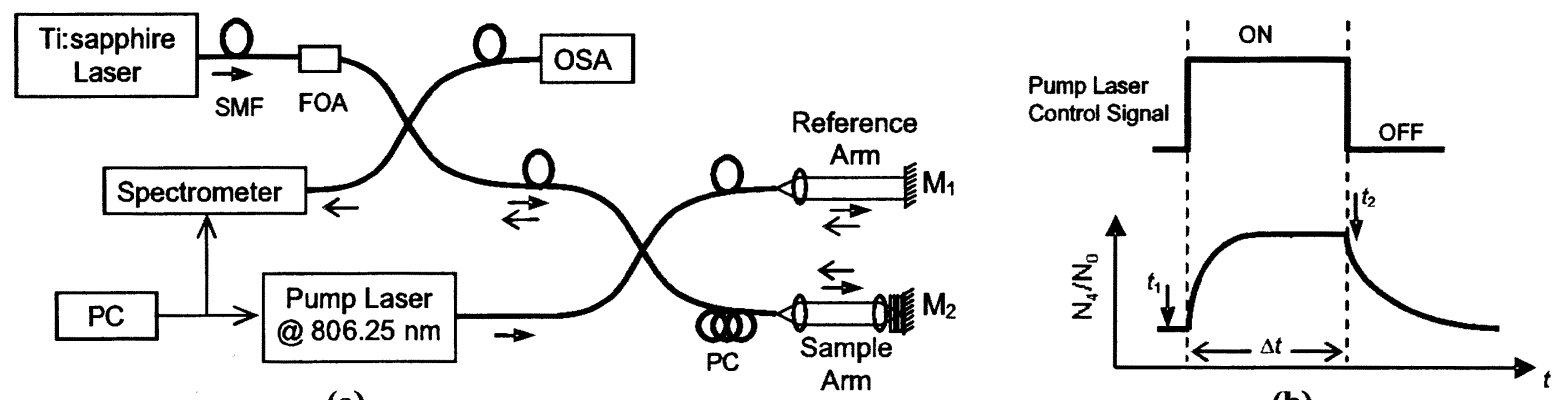

(a)

(b)

Fig. 3. (a) Proof- of- concept molecular contrast OCT setup; PC: Polarization controller, OSA: Optical spectrum analyzer, FOA: Fiber-optic attenuator, PC: Personal computer. (b) Timing diagram for the ICG-NaI pump-probe scheme.

Figure 4(b) shows the timing diagram for the ICG-based pump-probe scheme for the spectral domain OCT. Before the pump laser is turned ON, the first OCT A-scan is acquired at time $t_{1}$. After a short time, the pump laser is turned ON for $\Delta t$ duration. The labview routine takes the second A-scan at time $t_{2}$ after the pump laser has turned OFF. For OCT images with spatial resolution no less the coherence length of the broadband light source, the detected OCT signal for a specific depth and center wavelength is given by

$$
P_{\mathrm{det}}(z)=2 \sqrt{P_{R} P_{S} R_{S}} \exp \left\{-\int_{0}^{z} \mu\left(\lambda, z^{\prime}\right) d z^{\prime}\right\},
$$

where $\mu\left(\lambda, z^{\prime}\right)$ accounts for the loss of light due to absorption and scattering in the sample at depth $z^{\prime}, P_{R}$ and $P_{S}$ represent the light collected from the reference and sample arms, respectively, and $R_{S}$ is the reflectivity of the sample. For a single reflector at depth $l$, the difference signal of the images recorded at times $t_{1}$ and $t_{2}$ is given by

$$
S=2 \sqrt{P_{R} P_{S} R_{S}} e^{-2 \sigma N_{0} l}\left(e^{2 \sigma N_{4} l}-1\right) .
$$

Equation (5) estimates the MCOCT SNR as a function of power collected from the system and ICG-NaI population $\mathrm{N}_{4}$ in the excited state. For $17 \mathrm{~mW}$ pump laser output and $200 \mu \mathrm{W}$ broadband probe light reaching $200 \mu \mathrm{M}$ ICG solution, the system in Fig. 3(a) with base OCT SNR of $100 \mathrm{~dB}$ will have an MCOCT SNR of $\sim 11 \mathrm{~dB}$. Figure 4(b)(c) show the OCT A-scan of a $\sim 1 \mathrm{~mm}$ path length cuvette filled with $200 \mu \mathrm{M}$ ICG solution in $1 \%$ albumin before and after $\sim 9 \mathrm{~mW}$ pump beam exposure for $5 \mathrm{sec}$ duration. For comparison, the A-scan of the cuvette filled with water is also provided in Fig. 4(a). Both the A-scans in Fig. 4(a) and (c) yield the same path length of (1.497-0.118)/1.3= $1.06 \mathrm{~mm}$ between the inner faces 1 and 2 of the cuvette.
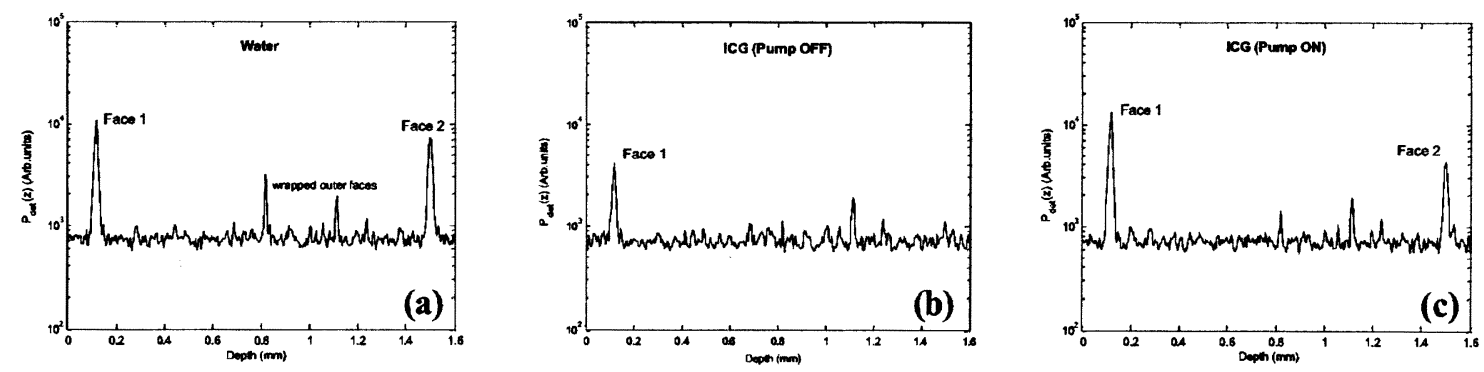

Fig. 4. OCT A-scans of $1 \mathrm{~mm}$ path length cuvette filled with (a) water and (b)-(c) ICG-NaI solution in $1 \%$ albumin; (b) and (c) show A-scans when the pump beam is OFF and ON, respectively.

In conclusion, we have shown for the first time that ICG-NaI, when mixed in a protein solution such as albumin or human plasma, can be used as a contrast agent in pump-probe scheme for molecular contrast OCT of biological samples. The demonstrated MCOCT scheme generates a difference image, revealing the distribution of ICG-NaI within the sample, from pairs of consecutive A-scans acquired before and after the pump wavelength illumination.

\section{References}

[1] M. R. Hee, J. A. Izatt, J. M. Jacobson, J. G. Fujimoto, E. A. Swanson, Optics Letters, 18(12), 950-952, (1993).

[2] J. A. Izatt, M. D. Kulkarni, S. Yazdanfar, J. K. Barton, and J. Welch, Optics Letters, 22(18), 1439-1441, September 1997.

[3] J. F. deBoer, T. E. Milner, M. J. C. van Gemert, J. S. Nelson, Optics Letters, Volume 22(12), 934-936, June 1997.

[4] K. D. Rao, M. A. Choma, S. Yazdanfar, A. M. Rollins, and J. A. Izatt, Optics Letters, 28(5), 340-342, March 2003.

[5] C. Yang, M. A. Choma, L. E. Lamb, J. D. Simon, and J. A. Izatt, Optics Letters, 29(12), 1396-1398, June 2004.

[6] C. Yang, L. E. L. McGukin, J. D. Simon, M. A. Choma, B. E. Applegate, and J. A. Izatt, Optics Letters, 29(17), $2016-2018$, Sep. 2004.

[7] H. Gratz, A. Penzkofer, C. Abels, R.-M. Szeimies, M. Landthaler, and W. Bäumler, J. Photochem. \& Photobiology, 128 (1999) $101-109$. 